\title{
Editorial briefing
}

\section{Changes and Challenges}

Welcome to this edition of HEX. This is the last 'catch-up' edition with a large volume of manuscripts published, which were submitted and accepted over the past 2 years. From the next edition, HEX is totally open access and authors will be pleased to see their manuscripts processed more speedily, thanks to the hard work of our editorial team.

You will notice the revised membership of the Editorial Board, and we are pleased to announce that we now have two members who bring the patient perspective to the Board. Fiona McKenzie started as the Patient Insight and Involvement Lead at UCL Partners (University College, London), and she has experience of publishing in peer-reviewed journals and in reviewing papers for publication. Fiona is a 'patient-turned-professional, and passionate about quality improvement and innovation in health care' and believes that involvement of patients and carters is key to this. John Maddison is a member of the Research User Group (RUG) at my institution, Keele University. The RUG is an integral part of the Research Institute, working with researchers at all stages of the research process. John has broad expertise in developing research questions with researchers, serving on Trial Steering Committees and advising about dissemination activities. $\mathrm{He}$ is also a member of the Patient Participation Group in his general practice. John accepted the position on the board to further the objective of
HEX in ensuring we meet our aims of focusing on public participation in health care and policy. John and Fiona will be invited to review submitted manuscripts, and, as with other members of the Editorial Board, their review may well decide the final decision we make on the manuscript.

We would also like to hear from 'patients' who would be willing to act as peer reviewers for submitted manuscripts. We can offer support from the editorial team, but also hope to establish a database of institutions that are willing to support their patient and public involvement and engagement (PPIE) groups in providing reviews of manuscripts.

We are also paying much more attention to the role played by patients in selecting and agreeing the research question, study design and methods, and interpretation and discussion of study findings, and in dissemination of results. The author's guidelines now refer to this explicitly.

Members of the editorial team realize that it is easy to make token gestures, but more challenging to achieve genuine partnership, whether in policymaking, clinical practice, service delivery or in research. We hope that we, at HEX, have moved a step closer to a genuine partnership with our patient readership and contributors.

We advise that if you are considering submitting a paper to HEX, please be explicit about the role patients have played, and the value that patients have added, to your research. 